\title{
Hard turning with variable micro-geometry PcBN tools
}

\author{
T. Özel ${ }^{\mathrm{a}, *}$, Y. Karpat ${ }^{\mathrm{b}}$, A. Srivastava (3) ${ }^{\mathrm{c}}$ \\ ${ }^{a}$ Department of Industrial and Systems Engineering, Rutgers University, NJ, USA \\ ${ }^{\mathrm{b}}$ Department of Industrial Engineering, Bilkent University, Ankara, Turkey \\ ${ }^{\mathrm{c}}$ TechSolve Inc., Cincinnati, OH, USA \\ Submitted by B. Kaftanoglu (1), Middle East Technical University, Ankara, Turkey
}

\section{A R T I C L E I N F O}

\section{Keywords:}

Hard machining

Tool

Edge geometry

\begin{abstract}
A B S T R A C T
This paper presents investigations on hard turning with variable edge design PcBN inserts. Turning of hardened AISI 4340 steel with uniform and variable edge design PcBN inserts is conducted, forces and tool wear are measured. 3D finite element modelling is utilized to predict chip formation, forces, temperatures and tool wear on uniform and variable edge micro-geometry tools. Predicted forces and tool wear contours are compared with experiments. The temperature distributions and tool wear contours demonstrate the advantages of variable edge micro-geometry design.
\end{abstract}

(C) 2008 CIRP.

\section{Introduction}

Hard turning is a more flexible, more environmentally benign and higher throughput alternative to grinding. However, process reliability and surface quality is still behind grinding [1]. In hard turning, polycrystalline cubic boron nitride (PcBN) cutting tools with various edge preparation (chamfer, radius, oval or waterfall edges, see Fig. 1) are preferred to protect the cutting edge from chipping $[1,2]$. Edge preparation must be carefully selected for a given application because it affects the surface integrity of the machined workpiece [3]. Heat generated during hard turning is also affected by edge preparation due to change in work material flow around the cutting edge. For example, a chamfered face provides excessive negative angle to the cutting action and results in high heat generation. PcBN tools rapidly wear out during hard turning at high cutting speeds mainly due to attained temperatures [4]. While a constant uncut chip thickness to edge radius ratio $\left(\lambda=t_{\mathrm{u}} / r_{\varepsilon}\right)$ should be maintained along the engagement area between PcBN insert and the workpiece, most edge preparations are applied uniformly all around the corner radius of the PcBN inserts. A uniform edge micro-geometry along the corner radius of the insert creates a very low edge radius to uncut chip thickness at the minor cutting edge. This causes more ploughing than shearing at the minor cutting edge resulting in excessive heat built-up and rapid wear. A variable edge microgeometry along the corner radius of the insert has the potential to reduce this heat built-up at the cutting edge enabling hard turning at higher cutting speeds and feeds with less tool wear [5] (see Fig. 2).

This paper aims to investigate the influence of variable edge micro-geometry insert edge design in hard turning both experimentally and via finite element analysis (FEA).

\footnotetext{
* Corresponding author.
}

\section{Variable micro-geometry edge design}

While uniform edge preparation strengthens tool cutting edge, it makes cutting less efficient especially when the ratio of uncut chip thickness to tool radius decreases. This is especially true when friction factor increases with decreasing uncut chip thickness to edge radius ratio. The work material is trapped near end of the uncut chip geometry along the corner radius. Inefficient cutting results in increased strains in the workpiece which in turn increases mechanical and thermal loads and results in high temperatures. Fig. 2 demonstrates the chip load of a uniform edge insert during cutting. The thickness of the chip varies from a maximum value, which is equal to feed $(f)$, to a minimum value on the tool's corner radius.

A CAD model of a variable edge design tool insert is given in Fig. 3 . It can be seen that edge radius at point $A$ is greater than that of at point $\mathrm{B}$ and $\mathrm{C}$. The edge radii at point $\mathrm{A}, \mathrm{B}$ and $\mathrm{C}$ are $r_{\varepsilon_{\mathrm{A}}}>r_{\varepsilon_{\mathrm{B}}}>r_{\mathrm{C}}$.

In Section A-A which is major (leading) cutting edge, uncut chip thickness is greater than the edge radius which indicates regular cutting. In Section B-B, at the end of the major (leading) edge, the uncut chip thickness is equal to the edge radius where the rubbing action becomes more dominant than shearing. In Section C-C, at the minor (trailing) cutting edge, the edge radius is larger than the thickness of the uncut chip and work material is rubbed against the workpiece. This rubbing action which results in increased temperatures on the tool and workpiece surfaces is believed to hinder the performance of the tool.

\section{Experimental procedure}

In this study, turning of annealed and hardened AISI 4340 steel (40 HRc) using PcBN inserts (50\% CBN + 40\% TiC + 6\% WC) with four different micro-geometries (uniform chamfer with $0.1 \mathrm{~mm}$ chamfer height and $20^{\circ}$ angle, uniform hone with $50 \mu \mathrm{m}$ (and $40 \mu \mathrm{m}$ ) edge radius, waterfall (WF) hone with $r_{\varepsilon}=30: 60 \mu \mathrm{m}$ radii, variable hone edge with $r_{\varepsilon_{\mathrm{A}}}=50 \mu \mathrm{m}, r_{\varepsilon_{\mathrm{B}}}=10 \mu \mathrm{m}$ radii) were considered. Turning 


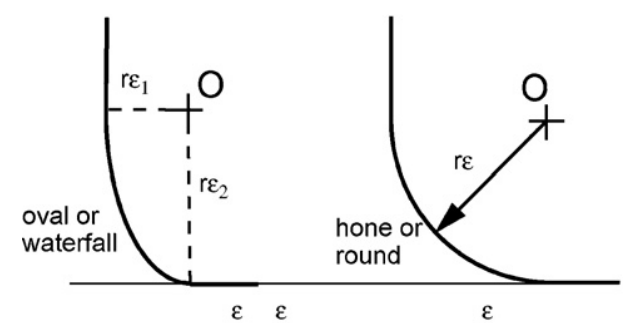

Fig. 1. Waterfall $\left(r_{\varepsilon_{1}}: r_{\varepsilon_{2}}\right)$ and round $\left(r_{\varepsilon}\right)$ hone micro-geometry edge design.

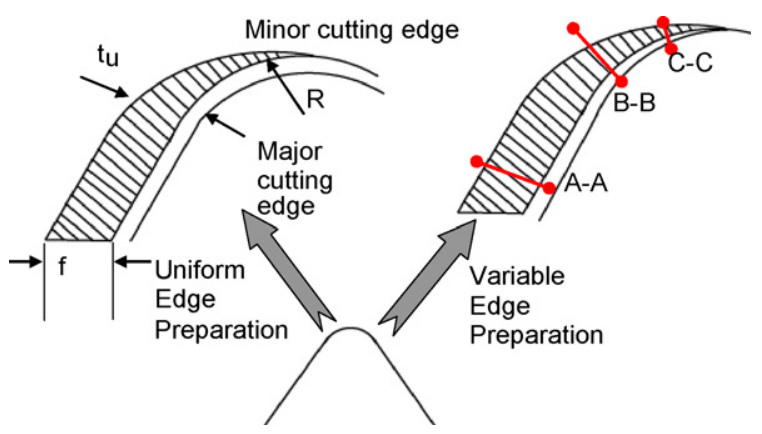

Fig. 2. Uniform vs. variable micro-geometry design.

experiments were conducted using a cylindrical bar specimen with a diameter of $71 \mathrm{~mm}$ and length of $305 \mathrm{~mm}$. Solid top PcBN inserts (TNG-423) were used with a MTGNR-123B right hand tool holder that provided $0^{\circ}$ lead, $-5^{\circ}$ side rake, and $-5^{\circ}$ back rake angles. The cutting forces were measured with a force dynamometer (Type 9121) mounted on the turret disk of the CNC lathe. It must be noted that the corner radius of the PcBN inserts (TNG-423) used in experiments is $R=1.2 \mathrm{~mm}$ which means that the cutting has been performed by the corner radius of the inserts $(d<R)$. The averages of the measured forces for each insert are shown in Fig. 4.

The effect of edge micro-geometry is apparent on measured radial (thrust) forces $\left(F_{\mathrm{t}}\right)$. For the cutting condition of $V=125 \mathrm{~m} /$ $\min , f=0.15 \mathrm{~mm} / \mathrm{rev}, d=1 \mathrm{~mm}$, waterfall hone edge with 30:60 $\mu \mathrm{m}$ edge radii yielded the lowest radial forces $\left(F_{\mathrm{t}}\right)$ followed by variable hone $50 \mu \mathrm{m}$ edge radius. The variable edge design produced higher tangential (cutting) forces $\left(F_{\mathrm{c}}\right)$ than other edge micro-geometry designs. Decreasing feed rate $(f=0.1 \mathrm{~mm} / \mathrm{rev})$ resulted in lower tangential forces. Largest radial (thrust) force was measured when turning with the chamfered insert whereas variable honed insert resulted in lowest radial force. These results may imply that more efficient cutting has been performed due to variable edge micro-geometry design that resulted in lower radial forces but slightly higher tangential forces.

Measured forces are fitted in log-linear models as function of cutting conditions and micro-geometry design parameters $\left(r_{\varepsilon_{1}}\right.$ and $\left.r \varepsilon_{2}\right)$. Thrust, feed and cutting force expressions are given in Eqs. (1)-(3) with R-sq =96.1, 99.5, and 99.8\%, respectively.

$$
\begin{aligned}
& F_{\mathrm{t}}=3789.5 d^{0.258} V^{-0.437} f^{0.466} r \varepsilon_{1}^{0.0829} r \varepsilon_{2}^{0.199} \\
& F_{\mathrm{z}}=2670.4 d^{1.14} V^{-0.327} f^{0.327} r \varepsilon_{1}^{0.0478} r \varepsilon_{2}^{0.112} \\
& F_{\mathrm{c}}=6568 d^{0.895} V^{-0.160} f^{0.695} r \varepsilon_{1}^{0.0145} r \varepsilon_{2}^{0.0053}
\end{aligned}
$$

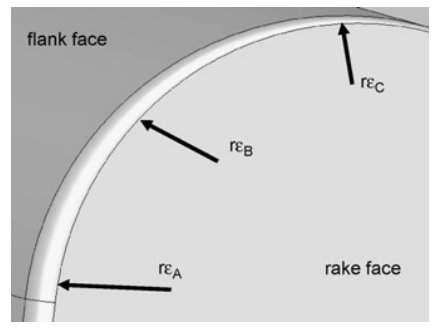

Fig. 3. CAD model of the variable hone edge design.

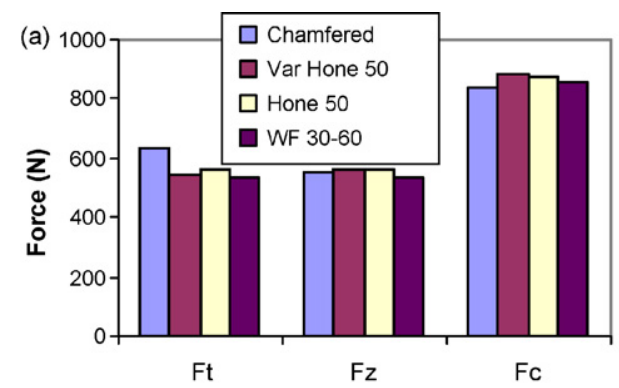

$V=125 \mathrm{~m} / \mathrm{min}, f=0.15 \mathrm{~mm} / \mathrm{rev}, d=1 \mathrm{~mm}$

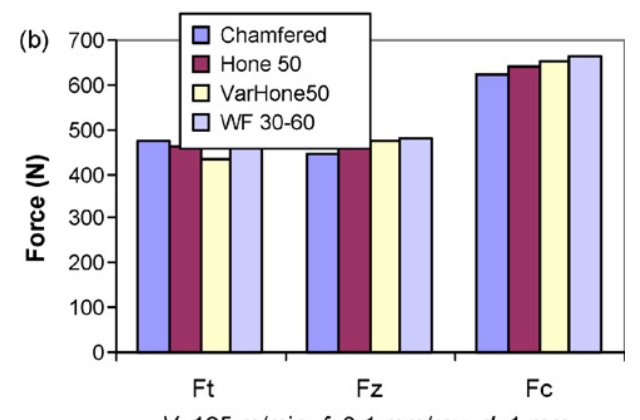

$V=125 \mathrm{~m} / \mathrm{min}, f=0.1 \mathrm{~mm} / \mathrm{rev}, d=1 \mathrm{~mm}$.

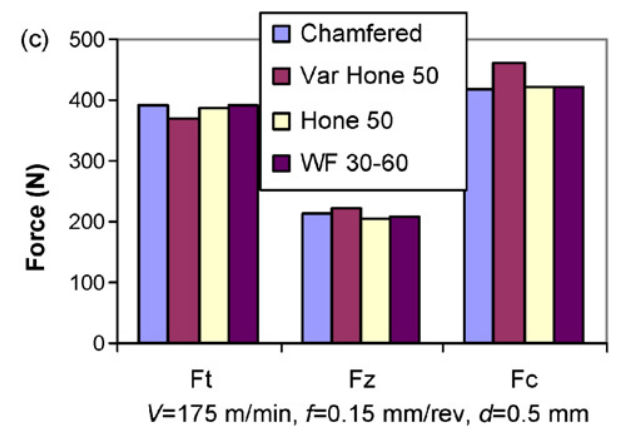

Fig. 4. Measured forces in turning of AISI 4340 steel.

\section{Finite element modeling of 3D turning}

Earlier finite element (FE) modelling studies have provided essential information about the influence of edge preparation on the process variables such as chip formation, forces, temperatures and stresses [6,7]. However, FE studies on 3D hard turning are very limited. Klocke and Kratz [4] utilized 3D FE modelling to investigate chamfer edge design in wiper inserts particularly identifying high temperature zones "hot-spots" on the tool face. More recently, Aurich and Bil [8] introduced 3D finite element modelling for segmented chip formation. In this study, a finite element modeling software (Deform 3D) is used to study the effects of uniform and variable micro-geometry edge design on process variables. This FEM software is based on an implicit Lagrangian computational routine with continuous adaptive remeshing. The workpiece is modeled as rigid-perfectly plastic material where the material constitutive model of this deformable body is represented with Johnson-Cook material model (see Eq. (4)) where $A=1504 \mathrm{MPa}, B=569 \mathrm{MPa}$, $n=0.22, C=0.003, m=0.9, T_{\text {melt }}=1426^{\circ} \mathrm{C}$ are the parameters for AISI 4340 steel as given by Gray et al. [9].

$\bar{\sigma}=\left[A+B(\bar{\varepsilon})^{n}\right]\left[1+C \ln \left(\frac{\dot{\bar{\varepsilon}}}{\dot{\bar{\varepsilon}}_{0}}\right)\right]\left[1-\left(\frac{T-T_{0}}{T_{\text {melt }}-T_{0}}\right)^{m}\right]$

The workpiece is represented by a curved model with $70 \mathrm{~mm}$ diameter which is consistent with the experimental conditions.

Table 1

Heat conduction coefficient

\begin{tabular}{lllrlll}
\hline$p[\mathrm{MPa}]$ & 0 & 30 & 180 & 300 & 420 & 600 \\
$h\left[\mathrm{~kW} \mathrm{~m}^{-2} \mathrm{~K}^{-1}\right]$ & 5 & 18 & 87 & 222 & 410 & 500 \\
\hline
\end{tabular}


Table 2

Thermomechanical properties of work and tool materials

\begin{tabular}{lcc}
\hline & AISI 4340 & PcBN \\
\hline Density $\left[\mathrm{kg} \mathrm{m}^{-3}\right.$ ] & 7850 & 4280 \\
Modulus of elasticity [GPa] & 205 & 587 \\
Poisson's ratio & 0.29 & 0.13 \\
Specific heat $\left[\mathrm{Jg}^{-1} \mathrm{~K}^{-1}\right]$ & 475 & 750 \\
Thermal conductivity [W m & $\left.44 \mathrm{~K}^{-1}\right]$ \\
Thermal expansion $\left[\mu \mathrm{m} / \mathrm{m} \mathrm{K}^{-1}\right.$ ] & 44.5 & 4.7 \\
\hline
\end{tabular}

Table 3

Pressure-dependent shear friction factor

\begin{tabular}{lcccrr}
\hline$p$ [MPa] & 500 & 1000 & 1500 & 2000 & 2500 \\
$m_{1}$ & 0.4 & 0.7 & 0.8 & 0.9 & 1.0 \\
$m_{2}$ & 0.2 & 0.35 & 0.4 & 0.5 & 0.7
\end{tabular}

Only a segment $\left(15^{\circ}\right)$ of the workpiece was modeled in order to keep the size of mesh elements small. Workpiece model includes 200,000 elements. The bottom surface of the workpiece is fixed in all directions. The cutting tool is modeled as a rigid body which moves at the specified cutting speed by using 125,000 elements. A very fine mesh density is defined at the tip of the tool and at the cutting zone to obtain fine process output distributions. The minimum element size for the workpiece and tool mesh was set to 0.025 and $0.009 \mathrm{~mm}$, respectively. Thermal boundary conditions are defined accordingly in order to allow heat transfer from workpiece to cutting tool. The heat conduction coefficient, $h$, is defined as a function of pressure as given in Table 1. Other thermomechanical properties are given in Table 2 .

All simulations were run at the same cutting condition ( $V=300 \mathrm{~m} / \mathrm{min}, f=0.15 \mathrm{~mm} / \mathrm{rev}, d=1 \mathrm{~mm})$. In 3D FE modeling, constant shear friction factor, $m$ and pressure-dependent shear friction factor, $m=f(p)$, have been benchmarked to identify the friction between micro-geometry tool and workpiece. Pressuredependent shear friction models used in simulations are given in Table 3.

FE simulation results for various friction values are given in Table 4. Pressure-dependent shear friction resulted in best force predictions (highlighted as bold). Especially, a reduced shear friction was observed in variable hone micro-geometry PcBN insert.

The comparison of experimental and simulated forces $\left(F_{\mathrm{c}}\right.$, cutting force, $F_{\mathrm{t}}$, thrust force and $F_{\mathrm{z}}$, feed force) are shown in Fig. 5. The simulated cutting forces are found to be in close agreements with the experimental ones.

Predicted temperature distributions on the tool are shown in Fig. 6 for three different micro-geometry inserts. These distributions depict that smallest hot zone formed on the variable honed tool and maximum temperatures of $853,724,664$ and $626^{\circ} \mathrm{C}$ were predicted for uniform chamfered, uniform waterfall (WF 30:60), uniform honed (Hone 40) and variable honed (Var. Hone 50) inserts, respectively. Due to the more uniform uncut chip thickness to the edge radius ratio along the variable micro-geometry insert, the cutting temperature at the same cutting condition is found to

Table 4

The effect of friction factor on process outputs

\begin{tabular}{|c|c|c|c|c|}
\hline$m$ & $F_{\mathrm{t}}[\mathrm{N}]$ & $F_{\mathrm{c}}[\mathrm{N}]$ & $F_{\mathrm{z}}[\mathrm{N}]$ & Tool $\left[{ }^{\circ} \mathrm{C}\right]$ \\
\hline \multicolumn{5}{|c|}{ Chamfer } \\
\hline 0.75 & 299 & 700 & 236 & 1020 \\
\hline$m_{1}$ & 386 & 775 & 335 & 853 \\
\hline \multicolumn{5}{|c|}{$\mathrm{WF}, r_{\varepsilon_{1}}=30 \mu \mathrm{m}, r_{\varepsilon_{2}}=60 \mu \mathrm{m}$} \\
\hline 0.3 & 342 & 782 & 225 & 836 \\
\hline$m_{1}$ & 360 & 690 & 310 & 724 \\
\hline \multicolumn{5}{|c|}{ Uniform Hone, $r_{\varepsilon}=40 \mu \mathrm{m}$} \\
\hline$m_{2}$ & 354 & 835 & 276 & 623 \\
\hline$m_{1}$ & 324 & 675 & 336 & 664 \\
\hline \multicolumn{5}{|c|}{ Var. Hone, $r_{\varepsilon_{\mathrm{A}}}=50, r_{\varepsilon_{\mathrm{B}}}=10 \mu \mathrm{m}$} \\
\hline$m_{1}$ & 390 & 800 & 352 & 693 \\
\hline$m_{2}$ & 285 & 690 & 258 & 638 \\
\hline
\end{tabular}

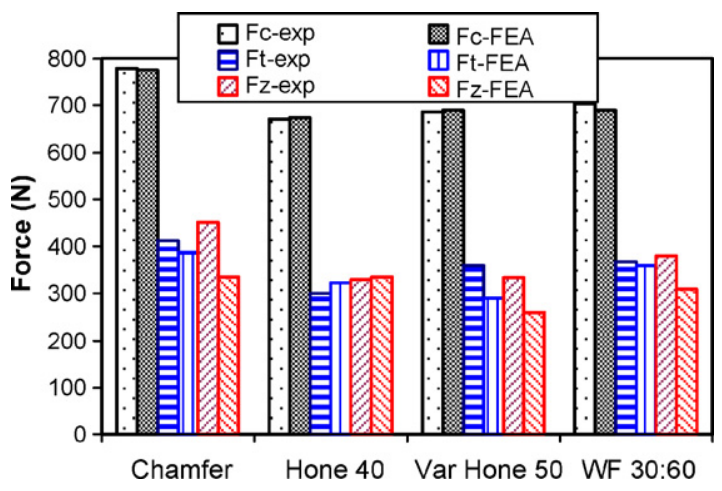

Fig. 5. Comparison of measured and simulated forces.

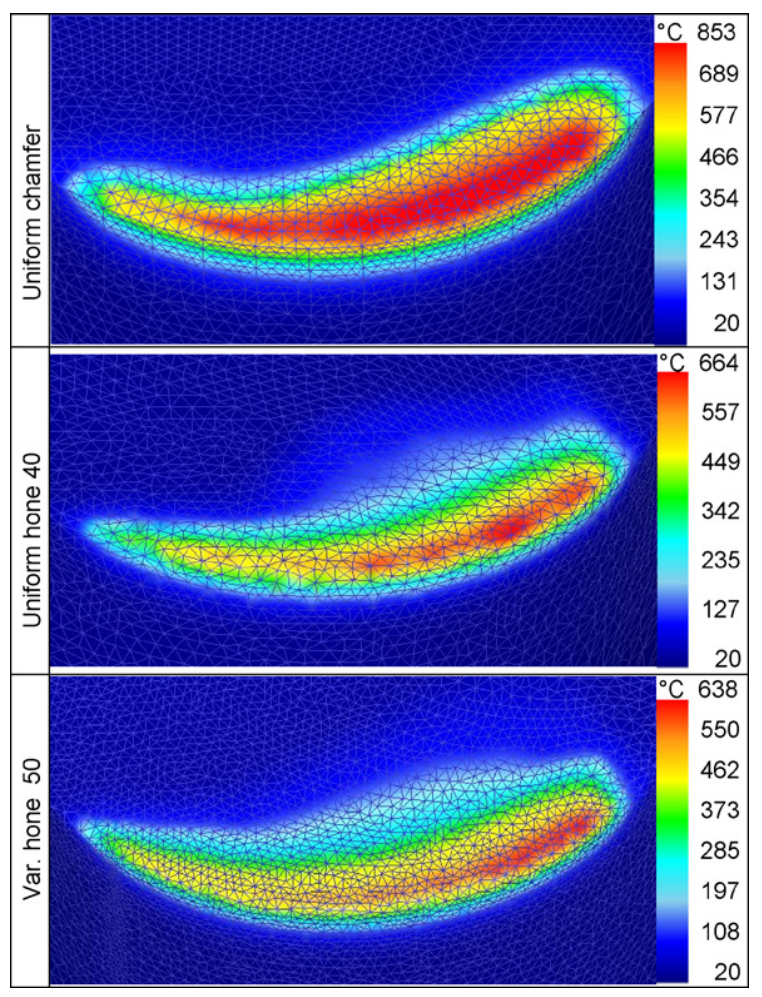

Fig. 6. Temperature distributions.

be significantly lower than other uniform micro-geometry (chamfered, honed and waterfall) inserts. In addition, the heat is seen more uniformly distributed along the cutting edge of the variable micro-geometry insert.

Predicted chip geometries and strain fields (see Fig. 7) indicate the effect of micro-geometry edge design on the plastic strain induced on the workpiece. Large edge micro-geometry insert has induced greater effective strain hence greater thermo-mechanical load to the workpiece. This creates higher heat generation and higher temperatures on the tool cutting edge.

Finite element simulations are also utilized to predict tool wear. The distributions of the process variables such as effective stresses, temperatures and sliding velocities allow the simulation of the tool wear on the tool rake and flank faces when combined with a tool wear model. The tool wear rate models describe the rate of volume loss on the tool rake and flank faces per unit area per unit time. There are many different tool wear rate models proposed in literature depending on the type of tool wear, i.e. adhesive, diffusive, etc. Among those, the tool wear rate model based on the adhesive wear proposed by Usui et al. [10], given in Eq. (5), uses interface temperature, normal stress, and sliding velocity at the contact surfaces as inputs and yields tool wear rate for a given location on the tool surface.

$\frac{\mathrm{d} V B}{\mathrm{~d} t}=c_{1} \sigma_{\mathrm{n}} V_{\mathrm{s}} \mathrm{e}^{\left(-c_{2} / T\right)}$ 


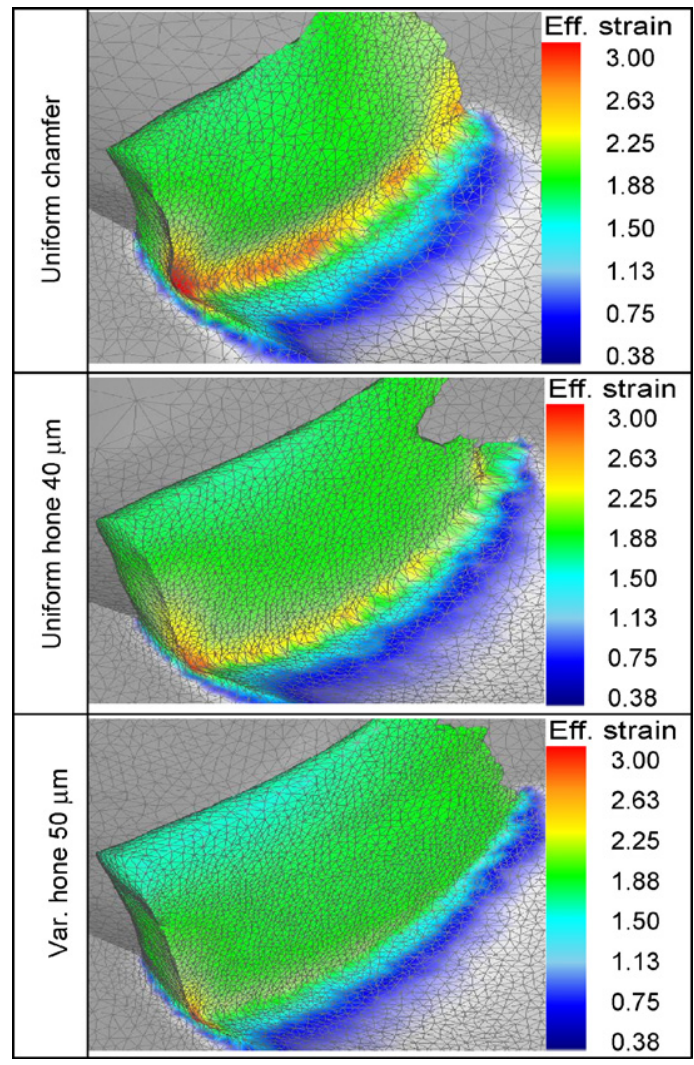

Fig. 7. Simulated chip formation and strain fields.

where $\mathrm{d} V B / \mathrm{d} t$ is the volumetric wear rate, $c_{1}$ and $c_{2}$ are the constants, $T$ is the temperature, $V_{\mathrm{s}}$ is the sliding velocity, and $\sigma_{\mathrm{n}}$ is the normal stress applied to the tool surface. In this study, the wear constants $c_{1}$ and $c_{2}$ for PcBN are found to be as $c_{1}=1 \times 10^{-6}$ and $c_{2}=0.9 \times 10^{2}$ by trial-and-error with FE simulations. Fig. 8 shows the simulated tool wear zones on the tool for uniform chamfer, uniform hone $r_{\varepsilon}=40 \mu \mathrm{m}$ and variable hone $r_{\varepsilon_{A}}=50 \mu \mathrm{m}$, $r_{\varepsilon_{\mathrm{C}}}=10 \mu \mathrm{m}$ inserts. Variable micro-geometry edge design has the lowest wear rate under the same cutting conditions.

\section{Conclusions}

In this paper, experimental and FE modelling investigations on hard turning with variable edge design PcBN inserts are presented. The results revealed that the variable edge preparation inserts perform better than uniform edge preparation counterparts if the variable edge is properly designed for the given cutting conditions. Specifically, the following conclusions can be made:

- Variable micro-geometry tool design reduces the heat generation along the tool cutting edge.

- Variable micro-geometry insert cutting edge induces less plastic strain on the machined workpiece.

- Tool wear is decreased with the use of a variable micro-geometry insert.

\section{Acknowledgement}

B. Kaftanoglu acknowledges support from Ministry of Industry and Trade-Turkey, SANTEZ: 00028.STZ.2007-1.

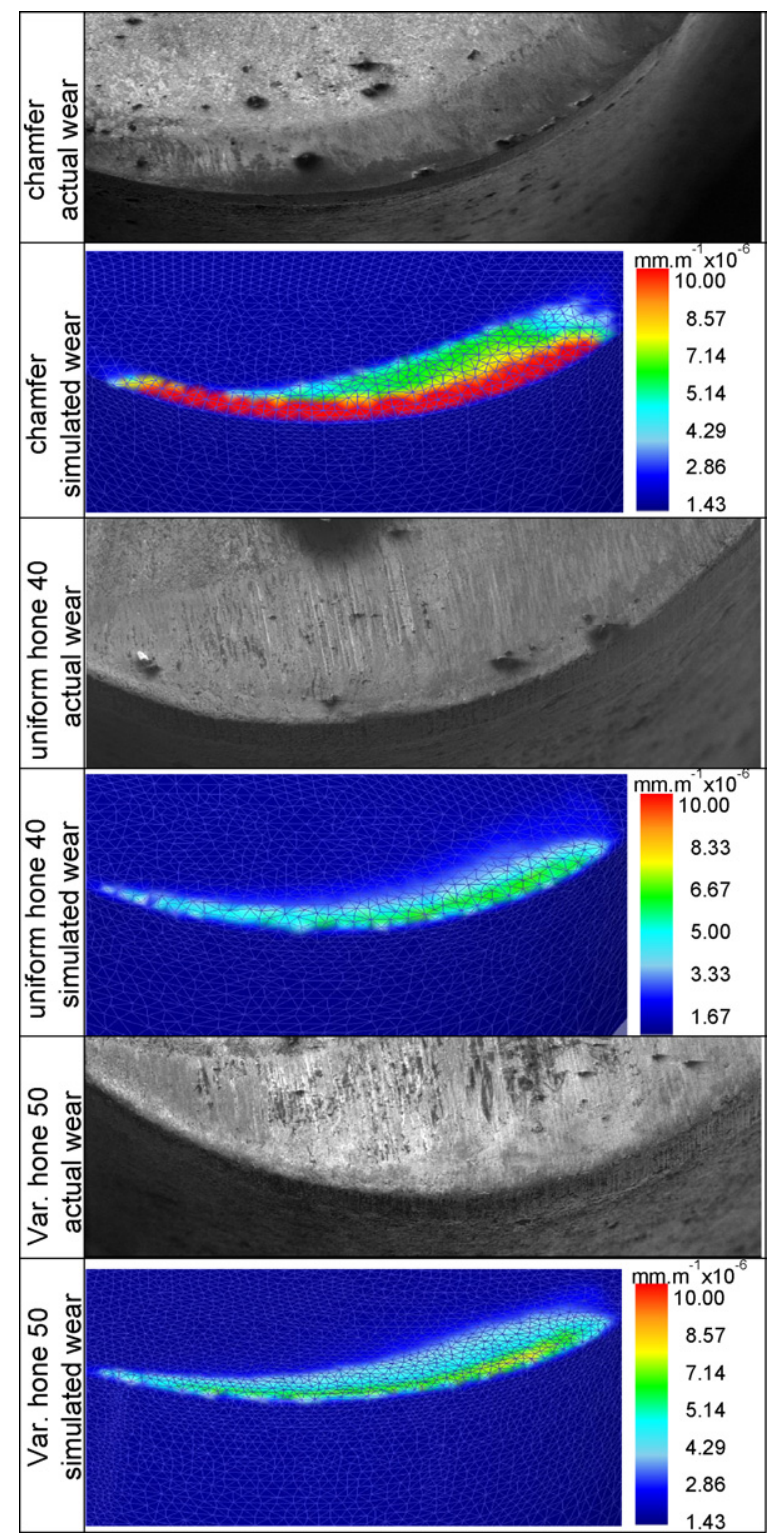

Fig. 8. Comparison of tool wear experiments with simulations.

\section{References}

[1] Klocke F, Brinksmeier E, Weinert K (2005) Capability Profile of Hard Cutting and Grinding Processes. Annals of the CIRP 54(2):557-580.

[2] Matsumoto Y, Hashimoto F, Lahoti G (1999) Surface Integrity Generated by Precision Hard Turning. Annals of the CIRP 48(1):59-62.

[3] Poulachon G, Moison A, Jawahir IS (2001) On Modeling Chip Formation During Hard Turning of 100Cr6 Bearing Steel. Annals of the CIRP 50(1):31-36.

[4] Klocke F, Kratz H (2005) Advanced Tool Edge Geometry for High Precision Hard Turning. Annals of the CIRP 54(1):47-50.

[5] Shaffer W (2000) Getting A Better Edge. Cutting Tool Engineering 52(3):44-48.

[6] Yen YC, Jain A, Altan T (2004) A Finite Element Analysis of Orthogonal Machining Using Different Tool Edge Geometry. Journal of Materials Processing Technology 146:72-81.

[7] Chen L, El Wardany TI, Nasr M, Elbestawi MA (2006) Effects of Edge Preparation and Feed When Hard Turning A Hot Work Die Steel With Polycrystalline Cubic Boron Nitride Tools. Annals of the CIRP 55(1):88-92.

[8] Aurich JC, Bil H (2006) 3D Finite Element Modelling of Segmented Chip Formation. Annals of the CIRP 55(1):47-50.

[9] Gray GT, Chen SR, Wright W, Lopez MF (1994) Constitutive Equations for Annealed Metals Under Compression at High Strain Rates and High Temperatures. Los Alamos Nat. Lab. LA-12699-MS.

[10] Usui E, Hirota A, Masuko M (1978) Analytical Prediction of 3D Cutting Process. Part 3. Cutting Temperature and Crater Wear of Carbide Tool. Journal of Engineering for Industry 100:236-243. 